\title{
Comments on the spontaneous strain and polarization of polycrystalline ferroelectric ceramics
}

\author{
Jiangyu Li and Kaushik Bhattacharya \\ Division of Engineering and Applied Science \\ California Institute of Technology, Pasadena, CA 91125
}

\begin{abstract}
A framework to calculate the spontaneous strain and polarization of a polycrystalline ferroelectric is presented, and various applications are discussed.
\end{abstract}

Keywords: Ferroelectric, spontaneous strain, texture, energy minimization

\section{INTRODUCTION}

It has recently been recognized that it is possible to obtain large strains through electrostriction of ferroelectric crystals obtained by polarization rotation ', or by domain switching as a result of applied electric field or mechanical stress ${ }^{2}$ However, large strains usually cannot be obtained in a polycrystalline ferroelectric ceramics due to the incompatibility between neighboring grains. So the identification of the optimal texture of polycrystals for high-strain actuation is worthwhile challenge. A related problem is the poling of the piezoelectric ceramic PZT, which is a solid solution of Lead Titanate and Lead Zirconate. The Titanium rich compositions where the material is tetragonal, and the rhombohedral rich compositions where the material is rhombohedral are both very difficult to pole, while the material is dramatically easy to pole at the 'morphotropic phase boundary'. An understanding of this difference is also desirable.

This work examines the macroscopic behaviors of ferroelectric solids in terms of the crystal systems and texture, using the homogenization theory and energy minimization following the framework used by Bhattacharya and Kohn ${ }^{3}$ to study polycrystalline shape-memory alloys. The spontaneous strain and polarization for single crystals is characterized, and the optimal texture for high-actuation strain for polycrystals is identified. The optimal electro-mechanical property of PZT at morphotropic phase boundary is also explained. This paper summarizes the results, and the reader is referred to $\mathrm{Li}$ and Bhaitacharya ${ }^{4}$ for detaiis.

\section{EFFECTIVE THEORY FOR FERROELECTRIC CERAMICS}

For a ferroelectric crystal $\Omega$ subject to an applied traction $\mathbf{t}_{0}$ on part of its boundary $\partial \Omega_{2}$, the displacement $\mathbf{u}$ and polarization $\mathbf{p}$ of the ferroelectric are those that minimize the potential energy

$$
\Phi(\mathbf{u}, \mathbf{p})=\int_{\Omega} \frac{1}{2} \nabla \mathbf{p} \cdot \mathbf{A} \nabla \mathbf{p}+W(\mathbf{x}, \mathbf{e}[\mathbf{u}], \mathbf{p})-\mathbf{E}_{0} \cdot \mathbf{p} d \mathbf{x}-\int_{\partial \Omega_{2}} \mathbf{t}_{0} \cdot \mathbf{u} d S+\int_{R^{3}} \frac{\varepsilon_{0}}{2}|\nabla \phi|^{2} d \mathbf{x}
$$

where the eiectric potential $\phi$ is determined by solving Maxwell equation in all space subject to appropriate boundary conditions, and $\mathbf{E}_{0}$ is the electric field in the absence of the ferroelectric. Briefly, the first term is the exchange energy or the energy needed to form domain walls, the second is the stored energy density which encodes the information about crystallography and texture, the third term represents the applied electric field, the fourth the applied mechanical loads and finally the last term is the electrostatic energy created due to the polarization distribution. Because of symmetry, the stored energy density exhibits a multi-well structure characterized by the set $\mathrm{K}=\cup_{i=1}^{K}\left\{\left(\mathbf{e}^{(i)}, \mathbf{p}^{(i)}\right)\right\}$ on which $W(\mathbf{x}, \mathbf{e}, \mathbf{p})=0$, where $\mathbf{e}^{(i)}$ is the transformation strain and $\mathbf{p}^{(i)}$ is the spontaneous polarization; see Fig. 1. It is first postulated that the exchange energy is negligible, and this is reasonable in large specimens. Second, a homogenization theory has been cieveloped for periodic ferroelectrics based on the theoretical framework of $\Gamma$-convergence ${ }^{4-6}$. This theory tells us that if the grain size is small compared to the size of the specimen, one may replace the spatially inhomogeneous term $W$ in the potential energy 
with an effective energy density $\bar{W}(\mathbf{e}, \mathbf{p})$, which are spatially homogeneous. $\bar{W}(\mathbf{e}, \mathbf{p})$ is the effective energy of a polycrystal when the average strain is $\mathbf{e}$ and the average polarization is $\mathbf{p}$. The theory also provides a formula for this effective energy; this formula is unfortunately not explicit but involves a minimization problem.

This effective energy $\bar{W}(\mathbf{e}, \mathbf{p})$ can be obtained in two steps; see Fig. 1. First, a mesoscopic energy $\hat{W}(\mathbf{e}, \mathbf{p})$ may be obtained for a single crystal, and then this can be homogenized to obtain the effective energy of the polycrystal. The mesoscopic energy is zero on a set $Z^{S}$ which identifies all the possible average spontaneous strain and polarization that a single crystal can display as it forms different domain patterns. The effective energy is zero on a set $Z^{P}$ which identifies all the possible average spontaneous strain and polarization that a polycrystalline ceramic can display as the grains form different domain patterns. The rest of the paper discusses estimates of these sets.

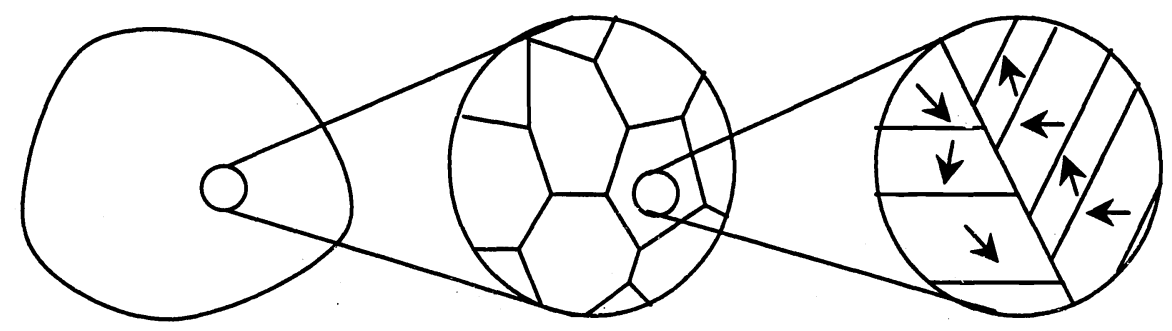

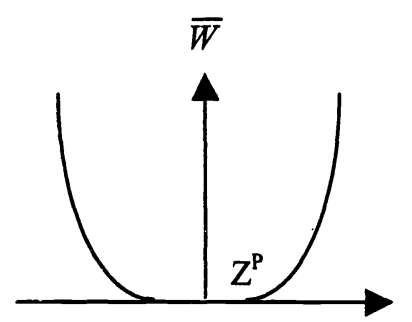

$(\mathbf{e}, \mathbf{p})$

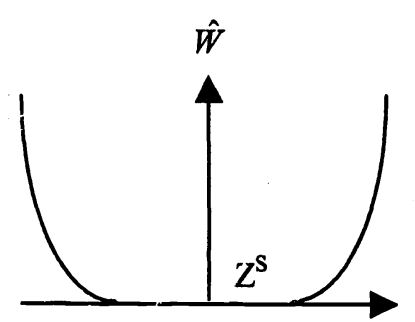

$(\mathbf{e}, \mathbf{p})$

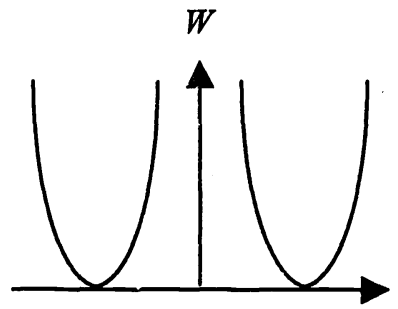

$(\mathbf{e}, \mathbf{p})$

Figure 1. The effective energy and zero energy set of single crystal and polycrystal.

\section{SPONTANEOUS STRAIN AND POLARIZATION}

The set $Z^{S}$ of spontatneous strains and polarizations of a single crystal correspond to the average strain and polarization as it forms different domain patterns. It has been shown ${ }^{4,7}$ that when the ferroelectric wells are pair-wise compatible, $\left(\mathbf{e}^{0}, \mathbf{p}^{0}\right)$ is a spontaneous strains and polarizations for a single crystal if and only if

$$
\mathbf{e}^{0}=\sum_{i=1}^{n} \lambda_{i} \mathbf{e}^{(i)}, \mathbf{p}^{0}=\sum_{i=1}^{n} \lambda_{i}\left(2 f_{i}-1\right) \mathbf{p}^{(i)},
$$

where $\lambda_{i} \geq 0$ and $\sum_{i=1}^{n} \lambda_{i}=1$, and $0 \leq f_{i} \leq 1$. In tetragonal crystal, the spontaneous strains of single crystal have fixed trace and zero off-diagonal elements. The magnitude of the diagonal elements is also bounded. These strains lie in a reduced 2dimensional strain space. In rhombohedral crystals, the spontaneous strain must have fixed and identical diagonal elements, and their off-diagonal elements are bounded. They lie in a reduced 3-dimensional strain space. In ferroelectric with tetragonal-rhombohedral morphotropic phase boundary (MPB), such as $\mathrm{PZT}$, or $\mathrm{Pb}\left(\mathrm{Zr}_{\mathrm{x}} \mathrm{Ti}_{1-\mathrm{x}}\right) \mathrm{O}_{3}$, around $\mathrm{x}=0.52$, the tetragonal and rhombohedral variants are compatible with each other if restriction on lattice parameters are satisfied, and the spontaneous strains in ferroelectric with MPB have fixed trace, and their off-diagonal elements are bounded. They lie in the 5-dimensional strain space. So crystal at MPB has much larger set of spontaneous strain than that of tetragonal and rhombohedral ferroelectrics. This has important implication on electro-mechanical properties of PZT ceramics. It is recently discovered that a previous unreported monoclinic phase exist in PZT at MPB ${ }^{8,9}$, where not all the variants are pair-wise compatible. We can derive an inner set of spontaneous strain, which lies in the 5-dimiensional strain space, with the trace fixed, and off-diagonal elements bounded. 
The set $Z^{\mathrm{P}}$ of spontatneous strains and polarizations of a polycrystal correspond to the average of locally varying but compatible spontaneous strain and polarization fields as the different grains form different microstructure. Thus the macroscopic spontaneous strain and polarization can be accommodated within each grain by the rearrangement of ferroelectric variants, and are compatible across grain boundaries. Unlike in the single crystal, the determination of the spontaneous strain depends on the polycrystal texture, and is generally very difficult. However, it is possible to estimate them from inside and outside using bounds. It is possible to show that the set $Z^{\mathrm{P}}$ contains a set $\mathrm{Z}^{\mathrm{T}}$ which is the intersection of the set $Z^{S}$ for each of the grains in the polycrystalline aggregate. This is the so-called Taylor bound, and it is based on the premise of uniform mesoscopic strain and polarization in each grain. Thus, it ignores the cooperative effects of grains, and is thus a conservative estimate. It is easy to calculate, and at times a surprisingly good indicator.

The Taylor bound has been used to give us the following conclusions. There are no macroscopic spontaneous strains in tetragonal materials, unless it has $<001>$ texture. Recall that the set $Z^{S}$ for the tetragonal materials is only two dimensional in strain space. Thus two grains will have serious mismatch unless they have very carefully chosen orientations. This carefully chosen orientation is exactly the $<001>$ texture; for all other textures there is incompatibility between grains resulting in no spontaneous strain. Similarly, there are no macroscopic spontaneous strain in rhombohedral materials unless it has a fiber texture. The situation is quite different, however, for ferroelectrics at the tetragonal-rhombohedral morphotropic phase boundary and also for monoclinic phases. Here a polycrystalline ceramic can have a macroscopic spontaneous strain independent of texture. Of course the extent of this will depend on the texture, but every polycrystal independent of texture has some. Recall that at both the MPB and monoclinic phase, the set $Z^{S}$ has an interior, and this allows the different grains have much more room to be compatible.

These observations explain the superior polability of PZT at compositions close to MPB, and also identify the textures for good electromechanical behavior in tetragonal and rhombohedral materials.

\section{REFERENCE}

1. Shrout, T. R. and Park, S. E., "Ultrahigh Strains and Piezoelectric Behavior in Relaxor Based Ferroelectric Single Crystals," J. Appl. Phys. 82, pp 1804-1811, 1997.

2. Burcsu, E., Ravichandran, G, and Bhattacharya, K, "Large Strain Electrostrictive Actuation in Barium Titanate," Appl. Phys. Lett. 77, pp. 1698-1700, 2000.

3. Bhattacharya, K and Kohn, R. V., "Elastic Energy Minimization and the Recoverable Strains of Polycrystalline ShapeMemory Materials," Arch. Ration. Mech. An. 139, pp. 99-180, 1997.

4. Li and Bhattacharya, "The Macroscopic Spontaneous Strain and Polarization of Ferroelectric Polycrystals," in preparation, 2001.

5. De Giorgi, E, "Sulla convergenza di alcune successioni d'integrali del tipo dell area," Rend. Matematica 8, pp. 277-294, 1975.

6. Muller, S., "Homogenization of Nonconvex Integral Functionals and Cellular Elastic Mateirlas," Arch. Ration. Mech. An. 99, pp. 189-212, 1987.

7. De Simone, A. and James, R. D., "A Constrained Theory of Magnetostriction," Preprint, 1998.

8. Noheda, B., Cox, D.E., Shirane, G., Gonzalo, J. A., Cross, L. E., and Park, S. E., "A Monoclinic Ferroelectric Phase in the $\mathrm{Pb}\left(\mathrm{Zr}_{1-\mathrm{x}} \mathrm{Ti}_{\mathrm{x}}\right) \mathrm{O}_{3}$ Solid Solution," Appl. Phys. Lett. 74, 2059-2061, 1999.

9. Noheda, B., Gonzalo, J. A., Cross, L. E., Guo, R., Park, S. E., Cox, D. E., and Shirane, G., "Tetragonal-to-Monoclinic Phase Transition in a Ferroelectric Perovskite: The Structure of $\mathrm{PbZr}_{0.52} \mathrm{Ti}_{0.48} \mathrm{O}_{3}$ " Phys. Rev. B61, pp. 8687-8695, 2000. 